\section{Klinische(r) Chemiker(in) (DGKL)}

\author{
A.M. Gressner ${ }^{1}$ und O.A. Gressner ${ }^{2}$ \\ ${ }^{1}$ Labor Dr. Wisplinghoff Berlin, Berlin, Deutschland \\ ${ }^{2}$ Labor Dr. Wisplinghoff Köln, Köln, Deutschland
}

Synonym(e) Anerkannte(r) Klinische Chemiker(in) der Deutschen Gesellschaft für Klinische Chemie und Laboratoriumsmedizin e.V.

\section{Englischer Begriff clinical chemist}

Definition Die sich über 5 Jahre erstreckende, strukturierte Weiterbildung der durch ein medizinisches oder geeignetes naturwissenschaftliches Eingangsstudium berechtigten Bewerber/Innen für das Fachgebiet Klinische Chemie ist eine satzungsgemäße Aufgabe der Deutschen Gesellschaft für Klinische Chemie und Laboratoriumsmedizin e.V. (DGKL; - Deutsche Gesellschaft für Klinische Chemie und Laboratoriumsmedizin e.V. (DGKL)), die für den Weiterzubildenden nach erfolgreicher Abschlussprüfung mit der Anerkennung (Qualifikation) als Klinische(r) Chemiker(in) endet.

Beschreibung Mit Bildung der Sektion Klinische Chemie in der Gesellschaft für Physiologische Chemie im Jahr 1955 wurden Richtlinien zu Ausbildung und Funktionen der Leiter eines klinisch-chemischen Labors erarbeitet, die als Grundlage für die „Anerkennung als Klinischer Chemiker“ dienten. Mit Gründung der Deutschen Gesellschaft für Klinische Chemie e.V. (DGKC; > Deutsche Gesellschaft für Klinische Chemie e.V. (DGKC)) im Jahr 1964 in Mosbach wurde die Weiterbildung zu einer zentralen, satzungsgemäßen Aufgabe der DGKC, die nach Fusion mit der Deutschen Gesellschaft für Laboratoriumsmedizin e.V. (DGLM; > Deutsche Gesellschaft für Laboratoriumsmedizin e.V. (DGLM)) zur DGKL e.V. am 08. Mai 2003 von dieser in die Satzung übernommen wurde. Richtliniendetails, Prüfungs- und Verfahrensordnungen zur Erteilung der Anerkennung als Klinischer Chemiker wurden wiederholt den wechselnden Gegebenheiten angepasst: Als abgeschlossene Eingangsstudiengänge zur Weiterbildung sind Humanmedizin (Staatsexamen) sowie Chemie, Biochemie oder Biologie, jeweils als Diplom oder Master of Science, zugelassen. Von der Weiterbildungszeit mit insgesamt 5 Jahren sind mindestens 4 Jahre unter der Leitung eines/ einer zur Weiterbildung durch die DGKL befugten Klinischen Chemikers/Klinischen Chemikerin in einem der Krankenversorgung dienenden klinisch-chemischen Laboratorium abzuleisten. $\mathrm{Zu}$ Beginn der Weiterbildung ist eine Anmeldung bei der Weiterbildungskommission erforderlich. Während der Weiterbildung wird eine wissenschaftliche Betätigung erwartet. Nach Durchsicht der erforderlichen Unterlagen erfolgt eine mündliche Abschlussprüfung, die bei Erfolg mit der Aushändigung der Urkunde endet. Für Details s. aktuelle Richtlinien in den Mitteilungen der Klinischen Chemie und auf der Homepage der DGKL (www.dgkl.de).

\section{Literatur}

Büttner J (2004) Kurze Chronologie der geschichtlichen Entwicklung der Deutschen Gesellschaft für Klinische Chemie e.V. bis zur Verschmelzung zur Deutschen Vereinten Gesellschaft für Klinische Chemie und Laboratoriumsmedizin e.V. Klinische Chemie Mitteilungen 35(5):89-95 